\title{
Efficiency at the maximum power of the power law dissipative Carnot-like Heat engines with non-adiabatic dissipation
}

\author{
M. Ponmurugan \\ Department of Physics, School of Basic and Applied Sciences, \\ Central University of Tamilnadu, Thiruvarur - 610 005, \\ Tamilnadu, India. e-mail:ponphy@cutn.ac.in
}

December 6, 2019

\begin{abstract}
We study the efficiency at the maximum power of non-adiabatic dissipative (internally dissipative friction in finite time adiabatic processes) Carnot-like heat engines operate in finite time under the power law dissipation regime. We find that the non-adiabatic dissipation does not influence the universal minimum and maximum bounds on the efficiency at the maximum power obtained in the generalized dissipative Carnot-like heat engines which does not take in to account the non-adiabatic dissipation.
\end{abstract}

Keywords: Heat engine, irreversible thermodynamics, power law dissipation, efficiency, universality

\section{Introduction}

Finite time thermodynamic studies of heat engines operate between hot and cold heat reservoirs focus mainly on improving its performance towards the traditional equilibrium Carnot engine [1. Carnot engine follows a particular cycle of infinite long duration called as the Carnot cycle, which consists of two isothermal and two adiabatic processes. The engine efficiency is defined as $\eta=W / Q_{h}$, where $W$ is the work performed and $Q_{h}$ is the amount of heat absorbed from the hot reservoir at a higher temperature $T_{h}$, while the engine delivers the heat $Q_{c}$ to the cold reservoir at temperature $T_{c}$. The efficiency of finite time heat engines are bounded below the Carnot engine efficiency, $\eta_{C}=1-T_{c} / T_{h}$. The power delivered by the cyclic heat engine is $P=W / \tau$, where $\tau$ is the total time taken to complete the given cycle.

There is a trade off between power and efficiency. The general belief is that $\eta_{C}$ can be achieved only at zero power. This has been proved for different systems working under different conditions, such as, thermoelectric classical and quantum systems [2, 3, micro and nano systems driven by periodic temperature variations [4, periodically driven system based on Onsager Coefficient [5], thermoelectric transport of quantum system 6 and master equation approach of classical and quantum systems $[7,8]$. While several other studies showed that $\eta_{C}$ 
also attained at nonzero power. Few of the studies for the thermoelectric system with broken time-reversal symmetry [9, 10, classical and quantum Carnot cycle at finite power 11, 12, efficiency statistics of heat devises 13, quantum Otto engine with finite-size scaling [14] and quantum Otto cycle of two-level system [15]. Studies based on sub-linear transport law [16], stochastic thermodynamics [17, 18], steady state heat engine [19], electrically charged black hole [20, classical harmonic oscillator under linear response regime [21] and trapped Bose gas in a quantum heat engine 22] showed that the system efficiency approaches $\eta_{C}$ at finite power. Recent studies based on classical Markov processes [23, 24, fluctuation of work and power [25, 26], exergy [27], quantum dot model with zero entropy production [28] and information engines [29, 30] also proved the attainability of $\eta_{C}$ at nonzero power.

The real heat engines operate in the finite time duration of non-zero power output with the efficiencies less than $\eta_{C}$. Different optimization procedures are introduced to enhance the performance of heat engines [31, 32, 33, 34]. In particular, the efficiency at maximum power, $\eta_{P}$, is being used frequently to investigate the improved performance of heat engines. This can be done by optimizing the efficiency of heat engines at maximum power condition. Earlier attempts to optimize the heat engine efficiency at maximum power was investigated by different researchers independently by employing different formulations, which are generally called as the Curzon-Ahlborn efficiency and is given by $\eta_{C A}=1-\sqrt{\frac{T_{c}}{T_{h}}}=1-\sqrt{1-\eta_{C}}$ [35, 36]. The efficiency at maximum power obtained from this formulation closely matches with the observed efficiencies of real heat engines [37. Nevertheless, these models does not provide the universal bounds on the efficiency at maximum power [1, 38, which is the central focus of the present work.

The phenomenological model of the finite time heat engine proposed to study the universal bounds on the efficient at maximum power is the low-dissipation Carnot engine [39, 40]. The study on the microscopic model of quantum heat engine indicated the presence of the power law like dissipation of Carnot-like heat engines [41]. The generalization of the low-dissipation model was discussed earlier [42] and also showed recently that [43] the power law dissipation incorporated in the model provides the generalized universal nature of lower bound, $\frac{\eta_{C}}{(\delta+1)}$, and the upper bound $\frac{\eta_{C}}{(\delta+1)-\delta \eta_{C}}$ on the efficiency at maximum power, where $\delta \geq 0$ is the degree of power law dissipation. The above results are derived under the assumption that the time taken to complete the adiabatic expansion and compression processes are negligible.

There are few studies on the low-dissipation model, which include the nonadiabatic dissipation in finite time adiabatic processes 44, 45, 46. The dissipation due to the effects of inner friction during the finite time adiabatic process is known as non-adiabatic dissipation [45, 47]. These studies showed that the non-adiabatic dissipative term additionally incorporated in the low-dissipation model does not influence the extreme bounds on the efficiency at maximum power [44, 45]. This raises a question whether such a non-adiabatic dissipative term can influence the universal bounds on the efficiency at maximum power of the power law dissipative Carnot-like heat engines? In order to answer this, in this paper, we study the efficiency at maximum power of non-adiabatic dissipative Carnot-like heat engines operate in a finite time under the power law dissipation regime [42, 43]. 


\section{Power law dissipation Carnot-like heat engine}

The power law dissipation Carnot-like heat engine model follows the cycle consisting of two isothermal processes of finite time duration and two instantaneous adiabatic processes. The working substance is in contact with the hot reservoir at temperature $T_{h}$ in the isothermal expansion and at temperature $T_{c}$ in the isothermal compression during the time interval $t_{h}$ and $t_{c}$, respectively. The amount of heat $Q_{h}$ and $Q_{c}$ exchanged between the hot and cold reservoirs and the working substance are modeled as [39, 42, 43]

$$
\begin{gathered}
Q_{h}=T_{h}\left\{\Delta S-\Delta S_{h}^{i r}\right\}, \\
Q_{c}=T_{c}\left\{-\Delta S-\Delta S_{c}^{i r}\right\},
\end{gathered}
$$

where $\Delta S_{i}^{i r}=\alpha_{i}\left(\frac{\sigma_{i}}{t_{i}}\right)^{\frac{1}{\delta}}, i: h, c$, are the irreversible entropy production, $\sigma_{i}=$ $\lambda_{i} \Sigma_{i}$, in which $\Sigma_{i}$ are the isothermal dissipation coefficients, $\lambda_{i} \& \alpha_{i}$ are the tuning parameters and $\pm \Delta S$ is the change in entropy of the working substance during isothermal expansion $(+)$ and compression $(-)$, which ensures that the system has zero change in total entropy for the cyclic process [39]. The $\delta \geq 0$ is a real number that represents the level of (power law) dissipation present in the system 43 . The system is in normal or low-dissipation regime with $\delta=1$, when it deviates below $(0<\delta<1)$ and above $(\delta>1)$ from the low-dissipation regime are called, respectively, sub and super dissipation regime [42. Since the efficiency obtained by the practical heat engines are not necessarily in the lowdissipation regime [37, our earlier results showed that the heat engines might also operate in the sub or super dissipation regime [43].

The recent experimental study validated $1 / \tau$ scaling of the irreversible entropy production in a finite-time isothermal process when the system undergoes a long contact time $\tau$ with the thermal bath [48]. Further, a recent theoretical study on quantum Otto engine showed (in terms of extra adiabatic work) $1 / \tau^{2}$ scaling of the irreversible entropy production in a finite-time adiabatic process [49]. Here $\tau$ is the controlling time of the long-time adiabatic process. The authors showed that the special control schemes of the finite-time adiabatic process improved the maximum power and the efficiency of the finite-time Otto engine.

In the case of including the non-adiabatic dissipation in our model, $Q_{c}$ contains additionally the irreversible entropy production $\Delta S_{a}^{i r}$ and $\Delta S_{b}^{i r}$, respectively, during the finite time adiabatic expansion and compression [44, 45, 46]. These entropy productions can also be assumed to obey the power law dissipation with respect to time for completing the adiabatic processes, which are of the form, $\Delta S_{j}^{i r}=\alpha_{j}\left(\frac{\sigma_{j}}{t_{j}}\right)^{\frac{1}{\delta}}, j: a, b$, where $\sigma_{j}=\lambda_{j} \Sigma_{j}$, in which $\Sigma_{j}$ are the adiabatic dissipation coefficients [44], $\lambda_{j} \& \alpha_{j}$ are the corresponding tuning parameters in the adiabatic process.

The amount of heat $Q_{c}$ exchanged between the cold reservoir and the working substance which contains the entropy productions due to non-adiabatic dissipations is given by [44, 45, 46]

$$
Q_{c}=T_{c}\left\{-\Delta S-\Delta S_{c}^{i r}-\Delta S_{a}^{i r}-\Delta S_{b}^{i r}\right\}
$$




$$
=T_{c}\left\{-\Delta S-\sum_{j=c, a, b} \Delta S_{j}^{i r}\right\}=T_{c}\left\{-\Delta S-\sum_{j=c, a, b} \alpha_{j}\left(\frac{\sigma_{j}}{t_{j}}\right)^{\frac{1}{\delta}}\right\} .
$$

Work performed by the engine during the total time period $\tau=t_{h}+t_{c}+t_{a}+t_{b}$ is $-W=Q_{h}+Q_{c}$. The convention used here is that the work and heat absorbed by the system are positive [39]. The power generated during the Carnot cycle is,

$$
P=\frac{-W}{\tau}=\frac{1}{\tau}\left\{\left(T_{h}-T_{c}\right) \Delta S-T_{h} \alpha_{h}\left(\frac{\sigma_{h}}{t_{h}}\right)^{\frac{1}{\delta}}-T_{c} \sum_{j=, c, a, b} \alpha_{j}\left(\frac{\sigma_{j}}{t_{j}}\right)^{\frac{1}{\delta}}\right\} .
$$

Using Eq.(1), $Q_{c}$ can be rewritten as

$$
Q_{c}=-T_{c}\left\{\frac{Q_{h}}{T_{h}}+\sum_{i=h, c, a, b} \alpha_{i}\left(\frac{\sigma_{i}}{t_{i}}\right)^{\frac{1}{\delta}}\right\} .
$$

Then,

$$
Q_{h}+Q_{c}=\eta_{C} Q_{h}-T_{c}\left\{\sum_{i=h, c, a, b} \alpha_{i}\left(\frac{\sigma_{i}}{t_{i}}\right)^{\frac{1}{\delta}}\right\}
$$

The engine efficiency during the Carnot cycle is

$$
\eta=\frac{Q_{h}+Q_{c}}{Q_{h}}=\eta_{C}-\frac{T_{c}}{Q_{h}}\left\{\sum_{i=h, c, a, b} \alpha_{i}\left(\frac{\sigma_{i}}{t_{i}}\right)^{\frac{1}{\delta}}\right\} .
$$

Using Eq.(1), the above equation can be rewritten as,

$$
\eta=\eta_{C}-\frac{T_{c}}{T_{h}\left[\frac{\Delta S}{\alpha_{h}}\left(\frac{t_{h}}{\sigma_{h}}\right)^{\frac{1}{\delta}}-1\right]}\left\{1+\sum_{j=c, a, b} \frac{\alpha_{j}}{\alpha_{h}}\left(\frac{\sigma_{j} t_{h}}{\sigma_{h} t_{j}}\right)^{\frac{1}{\delta}}\right\}
$$

and the power generated during the Carnot cycle is given by

$$
P=\frac{1}{\tau}\left\{\left(T_{h}-T_{c}\right) \Delta S-T_{h} \alpha_{h}\left(\frac{\sigma_{h}}{t_{h}}\right)^{\frac{1}{\delta}}-T_{c} \sum_{j=c, a, b} \alpha_{j}\left(\frac{\sigma_{j}}{t_{j}}\right)^{\frac{1}{\delta}}\right\} .
$$

The values of $t_{i}(i: h, c, a, b)$ at which the power becomes maximum are given by,

$$
\begin{aligned}
& t_{h}=\left\{\frac{\alpha_{h} T_{h} \sigma_{h}^{\frac{1}{\delta}}}{\left(T_{h}-T_{c}\right) \Delta S}\left(1+\frac{1}{\delta}\right)\left[1+\sum_{j=c, a, b}\left(\frac{\alpha_{j} T_{c}}{\alpha_{h} T_{h}}\right)^{\frac{\delta}{\delta+1}}\left(\frac{\sigma_{j}}{\sigma_{h}}\right)^{\frac{1}{\delta+1}}\right]\right\}^{\delta}, \\
& t_{c}=\left\{\frac{\alpha_{c} T_{c} \sigma_{c}^{\frac{1}{\delta}}}{\left(T_{h}-T_{c}\right) \Delta S}\left(1+\frac{1}{\delta}\right)\left[1+\left(\frac{\alpha_{h} T_{h}}{\alpha_{c} T_{c}}\right)^{\frac{\delta}{\delta+1}}\left(\frac{\sigma_{h}}{\sigma_{c}}\right)^{\frac{1}{\delta+1}}+\sum_{j=a, b}\left(\frac{\alpha_{j}}{\alpha_{c}}\right)^{\frac{\delta}{\delta+1}}\left(\frac{\sigma_{j}}{\sigma_{c}}\right)^{\frac{1}{\delta+1}}\right]\right\}^{\delta},
\end{aligned}
$$




$$
\begin{aligned}
& t_{a}=\left\{\frac{\alpha_{a} T_{c} \sigma_{a}^{\frac{1}{\delta}}}{\left(T_{h}-T_{c}\right) \Delta S}\left(1+\frac{1}{\delta}\right)\left[1+\left(\frac{\alpha_{h} T_{h}}{\alpha_{a} T_{c}}\right)^{\frac{\delta}{\delta+1}}\left(\frac{\sigma_{h}}{\sigma_{a}}\right)^{\frac{1}{\delta+1}}+\sum_{j=c, b}\left(\frac{\alpha_{j}}{\alpha_{a}}\right)^{\frac{\delta}{\delta+1}}\left(\frac{\sigma_{j}}{\sigma_{a}}\right)^{\frac{1}{\delta+1}}\right]\right\}^{\delta}, \\
& t_{b}=\left\{\frac{\alpha_{b} T_{c} \sigma_{b}^{\frac{1}{\delta}}}{\left(T_{h}-T_{c}\right) \Delta S}\left(1+\frac{1}{\delta}\right)\left[1+\left(\frac{\alpha_{h} T_{h}}{\alpha_{b} T_{c}}\right)^{\frac{\delta}{\delta+1}}\left(\frac{\sigma_{h}}{\sigma_{b}}\right)^{\frac{1}{\delta+1}}+\sum_{j=c, a}\left(\frac{\alpha_{j}}{\alpha_{b}}\right)^{\frac{\delta}{\delta+1}}\left(\frac{\sigma_{j}}{\sigma_{b}}\right)^{\frac{1}{\delta+1}}\right]\right\}^{\delta} .
\end{aligned}
$$

The ratio between $t_{j}(j: c, a, b)$ and $t_{h}$ which satisfies the following relation,

$$
\left(\frac{t_{j}}{t_{h}}\right)^{\frac{1}{\delta}+1}=\frac{\alpha_{j} T_{c}}{\alpha_{h} T_{h}}\left(\frac{\sigma_{j}}{\sigma_{h}}\right)^{\frac{1}{\delta}} .
$$

Combining Eqs. (8), 10) and (11), the efficiency at maximum power is obtained and is given below:

$$
\eta_{P}=\left(\frac{1}{\delta+1}\right) \frac{\eta_{C}}{1-\frac{\eta_{C}}{\left(1+\frac{1}{\delta}\right) \zeta}}
$$

where,

$$
\zeta=1+\varsigma\left(\frac{T_{c}}{T_{h}}\right)^{\frac{\delta}{\delta+1}}=1+\varsigma\left(1-\eta_{C}\right)^{\frac{\delta}{\delta+1}}
$$

and

$$
\varsigma=\sum_{j=c, a, b}\left(\frac{\alpha_{j}}{\alpha_{h}}\right)^{\frac{\delta}{\delta+1}}\left(\frac{\sigma_{j}}{\sigma_{h}}\right)^{\frac{1}{\delta+1}} .
$$

The above equation shows that $\eta_{P}$ in general does not exhibit any universal feature [50] and it depends only on the ratios of the individual parameters.

The power law dissipation of the phenomenological model with the nonadiabatic dissipation also governs the universal form of the efficiency under the assumption that the temperature difference between the two reservoirs is small, which is obtained by expanding $\eta_{P}$ in terms of $\eta_{C}$ as [43],

$$
\eta_{P}=\left(\frac{1}{\delta+1}\right) \eta_{C}+\frac{\delta}{(\delta+1)^{2}(1+\varsigma)} \eta_{C}^{2}+\frac{\delta^{2}}{(\delta+1)^{3}(1+\varsigma)^{2}} \eta_{C}^{3}+\ldots . .
$$

This shows that the non-adiabatic dissipation does not alter the super-universal feature of the efficiency at maximum power [43. This is the first main result of the present paper.

\section{Discussions}

If we neglect the inner friction, the adiabatic dissipation coefficients, $\sigma_{a} \rightarrow 0$ and $\sigma_{b} \rightarrow 0$, Eqs. (12) and (15) reduces to the earlier results of power law dissipation Carnot like heat engines with instantaneous adiabatic processes [43]. Now, we discuss whether the non-adiabatic dissipation will influence the universal nature of the extreme bounds on the efficiency at maximum power for different cases of symmetric and asymmetric dissipation limits. 
It is observed from Eq. (12) that the value of $\eta_{P}$ depends mainly on the term $\varsigma$ (Eq. (14)), which contains the ratios of dissipation coefficients and tuning parameters [43]. We obtain the minimum value of $\eta_{P}^{-}=\frac{1}{(\delta+1)} \eta_{C}$, when $\varsigma \rightarrow \infty$ and the maximum value of $\eta_{P}^{+}=\frac{\eta_{C}}{(\delta+1)-\delta \eta_{C}}$, when $\varsigma \rightarrow 0$. This shows that $\eta_{P}$ lies between these two extreme bounds, which is given by,

$$
\frac{1}{\delta+1} \eta_{C} \leq \eta_{P} \leq \frac{\eta_{C}}{(\delta+1)-\delta \eta_{C}}
$$

The lower and upper bounds can be obtained in the asymmetric dissipation limits of $\sigma_{h} \rightarrow 0$ and $\sigma_{h} \rightarrow \infty$, respectively, for any finite values of $\sigma_{j}(j$ : $c, a, b)$. Thus, one can obtain the generalized universal nature of lower and upper bounds on the efficiency at maximum power (Eq. 16p) under the combinations of isothermal and adiabatic asymmetric dissipation limits, which is same as the one obtained for power law dissipation Carnot-like heat engine model without having non-adiabatic disspation [42, 43. This is the second main result of the preent paper.

In the case of completely symmetric dissipation, $\sigma_{c}=\sigma_{h}=\sigma_{a}=\sigma_{b}$, the efficiency at maximum power $(\mathrm{Eq}, 12$ becomes,

$$
\eta_{P}^{s}=\frac{\eta_{C}}{(\delta+1)-\frac{\delta \eta_{C}}{\zeta_{s}}}
$$

where

$$
\zeta_{s}=1+\sum_{j=c, a, b}\left(\frac{\alpha_{j} T_{c}}{\alpha_{h} T_{h}}\right)^{\frac{\delta}{\delta+1}}
$$

Under the tuning condition, $\frac{\alpha_{j}}{\alpha_{h}}=\frac{1}{3} \frac{T_{h}}{T_{c}}, \eta_{P}^{s}$ reduces to the efficiency at maximum power of the stochastic heat engine [51] with $\delta=1$.

Finally, in order to see the ranges of $\varsigma$ and $\delta$ in which $\eta_{P}$ covers the observed efficiencies of different thermal power plants [39], we plotted $\eta_{P}$ as a function of $\eta_{C}$ in Figure 1 for different values of $\varsigma$ and $\delta$. The observed efficiencies of the various thermal power plants are represented in circles [37, 39, 52] and the solid line represents $\eta_{C A}$. The figure shows $\eta_{P}$ encompasses $\eta_{C A}$ and the observed efficiencies of different power plants, which are in the combinations of isothermal and non-adiabatic dissipations range $0 \leq \varsigma \leq 4$ with the power law dissipation range $0.6 \leq \delta \leq 1.75$.

\section{Conclusion}

We calculated the efficiency at the maximum power of the power law dissipation Carnot-like heat engines which taken in to account the non-adiabatic dissipation of finite time adiabatic processes. We found that the presence of non-adiabatic dissipation does not influence the universal feature of the generalized extreme bounds on the efficiency at maximum power. We expanded $\eta_{P}$ in terms of $\eta_{C}$ and also obtained the same universal form of the efficiency at maximum power. 


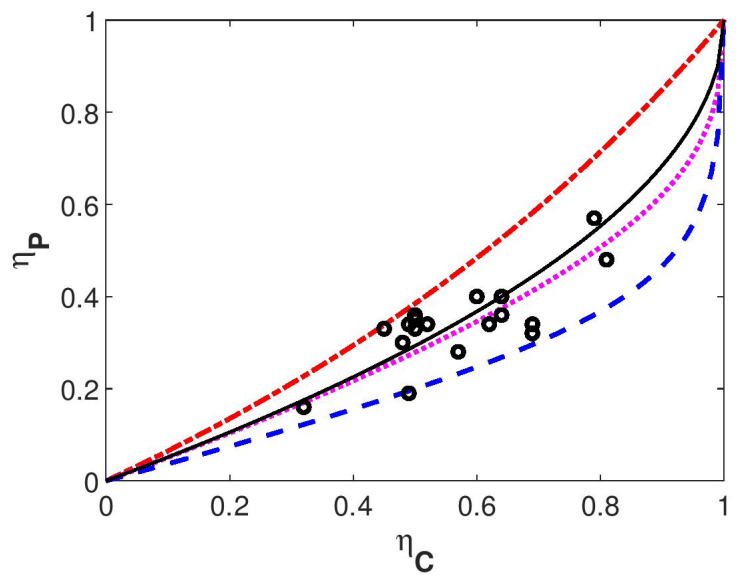

Figure 1: Efficiency at maximum power $\eta_{P}$ plotted as a function of $\eta_{C}$ for different values of $\delta$ and $\varsigma$. Top (dot-dashed line): for $\delta=0.6, \varsigma=0$, Middle (dotted line): $\delta=1$ and $\varsigma=2$ and bottom (dashed line): $\delta=1.75, \varsigma=4$. The observed efficiencies of the various thermal power plants are shown in circles [37, 39, 52. Solid line represents $\eta_{C A}$.

\section{References}

[1] R. S. Berry, V. A. Kazakov, S. Sieniutyez, Z. Szwast and A. M. Tsirlin, in Thermodynamics optimization of Finite-Time process (John Wiley and Sons, Chichester, 2000).

[2] K. Brandner and U. Seifert, Phys. Rev. E 91, 012121 (2015).

[3] K. Brandner and U. Seifert, Phys. Rev. E 93, 062134 (2016).

[4] K. Brandner, K. Saito and U. Seifert, Phys. Rev. X 5, 031019 (2015).

[5] K. Proesmans and C. Van den Broeck, Phys. Rev. Lett. 115, 090601 (2015).

[6] K. Yamamoto, O. Entin-Wohlman, A. Aharony, and N. Hatano Phys. Rev. B (R) 94, 121402 (2016).

[7] N. Shiraishi, K. Saito and H. Tasaki, Phys. Rev. Lett. 117, 190601 (2016).

[8] N. Shiraishi and H. Tajima, Phys. Rev. E 96, 022138 (2017).

[9] G. Benenti, K. Saito and G. Casati, Phys. Rev. Lett. 106, 230602 (2011).

[10] K. Brandner, K. Saito and U. Seifert, Phys. Rev. Lett. 110, 070603 (2013).

[11] A. E. Allahverdyan, K. V. Hovhannisyan, A. V. Melkikh and S. G. Gevorkian, Phys. Rev. Lett. 111, 050601 (2013).

[12] V. Holubec and A. Ryabov, Phys. Rev. E 96, 062107 (2017). 
[13] M. Polettini, G. Verley and M. Esposito, Phys. Rev. Lett. 114, 050601 (2015).

[14] M. Campisi and R. Fazio, Nat.Commun. 7, 11895 (2016).

[15] B. Leggio and M. Antezza, Phys. Rev. E 93, 022122 (2016).

[16] J. Koning and J. O. Indekeu, Eur. Phys. J. B 89, 248 (2016).

[17] M. Polettini and M. Esposito, Europhysics Letters, 118, 40003 (2017).

[18] J. S. Lee, H. Park, Scientific Reports 7, 10725 (2017).

[19] P. Pietzonka and U. Seifert, Phys. Rev. Lett. 120, 190602 (2018).

[20] C. V. Johnson, Phys. Rev. D 98, 026008 (2018).

[21] M. V. S. Bonanca, arXiv:cond-mat, 1809.09163 (2018).

[22] J. Wang, J. He, and Yong-li Ma, arXiv:cond-mat, 1803.03734 (2018).

[23] N. Shiraishi, Phys. Rev. E 95, 052128 (2017).

[24] N. Shiraishi, K. Saito, J. Stat. Phys. 174, 433 (2019).

[25] V. Holubec and A. Ryabov, Phys. Rev. E 96, 030102(R) (2017).

[26] V. Holubec and A. Ryabov, Phys. Rev. Lett. 121, 120601 (2018).

[27] M. Ponmurugan, J. Non-Equilib. Thermodyn. 44, 143 (2019).

[28] J. S. Lee, S. H. Lee, J. Um and H. Park, arXiv:1812.10651 (2019).

[29] M. Bauer, D. Abreu, and U. Seifert, J. Physics A: Math.Theor. 45, 162001 (2012).

[30] S. Rana and A. M. Jayannavar, J. Stat. Mech. 103207 (2016).

[31] C. Van den Broeck, Phys. Rev. Lett. 95, 190602 (2005).

[32] J. W. Stucki, Eur. J. Biochem. 109, 269 (1980).

[33] N. Sanchez-Salas, L. Lopez-Palacios, S. Velasco and A. C. Hernandez, Phys. Rev. E 82, 051101 (2010).

[34] Y. Zhang, J. Guo, G. Lin and J. Chen, J. Non-Equilib. Thermodyn. 42, 253 (2017); S. L. Medina and L. A. A. Hernandez, arXiv:1908.11861 (2019).

[35] I. I. Novikov, J. Nucl. Energy 7, 125 (1958).

[36] F. L. Curzon and B. Ahlborn, Am. J. Phys. 43, 22 (1975).

[37] S. L. Medina, G. V. Ortega and L. A. A. Hernandez, arXiv:1812.09350 (2018) and references therein.

[38] K. H. Hoffmann, J. M. Burzler and S. Schubert, J. Non-Equilib. Thermodyn. 22, 311 (1997) 
[39] M. Esposito, R. Kawai, K. Lindenberg and C. Van den Broeck, Phys. Rev. Lett. 105, 150603 (2010).

[40] J. Guo, J. Wang, Y. Wang and J. Chen, Phys. Rev. E 87, 012133 (2013).

[41] V. Cavina, A. Mari and V. Giovannetti, Phys. Rev. Lett. 119, 050601 (2017).

[42] W. Yang and T. Zhan-Chun, Commun. Theor. Phys. 59, 175 (2013).

[43] M. Ponmurugan, J. Stat. Mech. 113202 (2019).

[44] J. Wang and J. He, Phys. Rev. E 86, 051112 (2012).

[45] J. Wang, J. He and Z. Wu, Phys. Rev. E 85, 031145 (2012).

[46] Y. Hu et.al, Phys. Rev. E 88, 062115 (2013).

[47] T. Feldmann and R. Kosloff, Phys. Rev. E 61, 4774 (2000).

[48] Y. H. Ma, R. X. Zhai, C. P. Sun and H. Dong, arXiv: 1910.13434.

[49] J. F. Chen, C. P. Sun and H. Dong, Phys. Rev. E 100, 032144 (2019); arXiv: 1904.12128.

[50] U. Seifert, Phys. Rev. Lett. 106, 020601 (2011); S. H. Lee, J. Um and H. Park, Phys. Rev. E 98, 052137 (2018).

[51] T. Schmiedl and U. Seifert, Europhys. Lett. 81, 20003 (2008).

[52] R. S. Johal, Eur. Phys. J. Special Topics, 226, 489 (2017). 